\title{
Empoderamiento de mujeres y ventas online de vestimentas con esencia Shipibas un nuevo concepto del Free Shipibo Style, el caso de la Comunidad Shipibo-Konibo, Lima
}

\section{Empowerment of women and online sales of clothing with Shipibo essence, a new concept of Free Shipibo Style, the case of the Shipibo-Konibo Community, Lima}

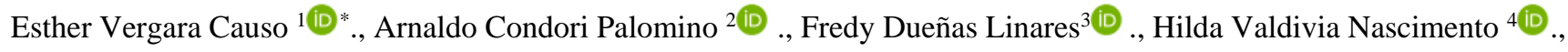

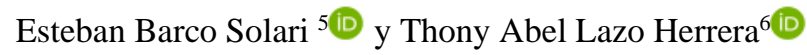

\section{RESUMEN}

La investigación se basa en las estrategias de marketing que deben emplear las mujeres Shipibo-Konibo en la venta de vestimentas con esencia Shipiba mediante las redes sociales: Facebook, Youtube, wordpress, Instagram, posicionándolas en el mercado online, en tiempos de la Covid-19. El estudio describe los impactos socioeconómicos que ha tenido la comunidad Shipiba en la implementación de estrategias de marketing para vender vestimentas oriundas de una cultura amazónica. El estudio utiliza una metodología cualitativa de diseño fenomenológico. La comunidad Shipibo-Konibo ha logrado entrar en la nueva concepción del Free Shipibo Style, una nueva tendencia de la moda actual, que ha logrado fusionarse con rasgos urbanos sin perder la esencia Shipiba. Se concluye que para ampliar el mercado es necesario la implementación de estrategias de potencialización de competencias basadas en aptitudes naturales de los miembros de las familias como unidad básica del empoderamiento.

Palabras clave: Marketing, ventas online, simbiosis cultural.

\begin{abstract}
The research is based on the marketing strategies to be used by Shipibo-Konibo women in the sale of Shipiba clothing through social networks: Facebook, Youtube, wordpress, Instagram, positioning them in the online market, in times of Covid-19. The study describes the socio-economic impacts that the Shipiba community has had in the implementation of marketing strategies to sell garments originating from an Amazonian culture. The study uses a qualitative phenomenological design methodology. The Shipibo-Konibo community has managed to enter the new concept of Free Shipibo Style, a new trend in current fashion, which has managed to merge with urban features without losing the Shipiba essence. It is concluded that in order to expand the market, it is necessary to implement strategies of empowerment based on the natural aptitudes of the members of the families as the basic unit of empowerment.
\end{abstract}

Keywords: Marketing, online sales, cultural symbiosis.

DOI: https://doi.org/10.37787/pakamuros-unj.v9i3.218

Recibido: 27/05/2021. Aceptado: 03/07/2021

* Autor para correspondencia

1. Universidad César Vallejo, Lima, Perú. Correo: esvergarac@ucvvirtual.edu.pe

2. Universidad Nacional del Altiplano, Puno, Perú. Correo: arnaldopelussa@gmail.com

3. Universidad Nacional Amazónica de Madre de Dios, Puerto Maldonado, Perú. Correo: fduenas@unamad.edu.pe

4. Universidad Nacional Mayor de San Marcos, Lima, Perú. Correo: hilda.valdivia@unmsml.edu.pe

5. Universidad César Vallejo, Lima, Perú. Correo: ebarcos@ucvvirtual.edu.pe

6. Universidad Nacional Amazónica de Madre de Dios, Puerto Maldonado, Perú. Correo: tlazo@unamad.edu.pe 


\section{INTRODUCCIÓN}

El impacto socioeconómico generado por la coyuntura de pandemia mundial ha tenido efectos colaterales en la ciudadanía (Li et al., 2020; Guevara et al., 2020). Un sector particular es el de las mujeres, quienes luego de un impacto de salubridad han tenido que buscar alternativas de emprendimiento para poder sobrevivir en tiempos difíciles. En ese sentido, se ha observado iniciativas propicias e las mujeres Shipibo-Konibo en la venta de artesanía, telares y manualidades mediante las redes sociales: Facebook, Youtube, wordpress, Instagram, posicionándolas en el mercado online, más aún en estos tiempos de la COVID-19, empleando estrategias de gestión de conocimiento empresarial que deben emplear, ya sea porque les capacitaron o porque lo aprendieron de manera mimética. En algunos estudios se ha demostrado que empodera a 3,749 mujeres, integrantes de 102 organizaciones regionales como Guatemala, El Salvador, México y Nicaragua (Ramos, 2016). Las mujeres participantes ganaron dinero, aumentaron su riqueza y mejoraron su capacidad para vender productos. Al mismo tiempo, acortó el tiempo de inversión, amplió su conocimiento de los derechos humanos, desarrolló su voz y liderazgo, y agregó autodesarrollo al equilibrio local de poder y la capacidad de influir en su imaginación. Empoderar a las mujeres que viven en los suburbios de las grandes ciudades con procesos comerciales son buenas lecciones y métodos (Machicado, 2016).

Desde luego, en la nueva tendencia de mercado los productos son valorizados a medida que son consumidos, por tanto, la gestión del conocimiento utiliza tecnología de punta y a la vanguardia junto con la comunicación para mejorar la competitividad de las personas. Ante esto, es necesario tomar nuevos enfoques: sistemático, participativo, interdisciplinario, proactivo, proceso y de competencias (Alles, 2008; Hernández y Pulido, 2011). Las Tecnologías de la Información y la Comunicación (NTIC) indujeron la amplitud de objetivos, el conocimiento de computadoras, redes, internet, intranets, correo electrónico, comercio electrónico, e-learning, portales y sitios web son las categorías mencionadas en la NTIC actual y la situación actual (Hernández y Pulido, 2011).

Se toma en consideración sus actividades de artesanía típicas de la comunidad Shipibo-Konibo de Cantagallo, sus telares coloridos, mantas de abrigo, sus mascarillas de tela, mediante las plataformas virtuales de las redes sociales digitales: Facebook, Youtube, Wordpress, Instagram y Whatsapp, promoviendo de esta manera mayores oportunidades en el mercado laboral online. Por otro lado, estas mujeres solían trasladarse a las calles de la ciudad o a mercados informales, siendo esto uno de los factores de mayor peligro para las familias en tiempos de COVID-19 (Vértiz-Osores et al., 2020). Es así que, el manejo de los softwares permite un mayor acceso a los mercados internacionales y el 
reconocimiento cultural de sus tradiciones ancestrales (Espinoza, 2016). Es importante estudiar el empoderamiento de las mujeres Shipibo-Konibo y las ventas online como una oportunidad, como un modelo de responsabilidad social en beneficio de la población peruana, sobre todo en aquellos que, hace décadas, dejaron los valles del río Ucayali para viajar a la capital peruana persiguiendo oportunidades en la gran ciudad (Briceño, 2020; Vergara, et al. 2021).

Para Villagomez (2019) los impactos positivos de las ventas atraen más a las mujeres que a los varones. Por otro lado, Cruz (2015) confirmó la satisfacción laboral de las mujeres en trabajos de costura debido a mejores ingresos. Briceño (2020) reconoció que las manifestaciones culturales de los grupos nativos han asimilado las costumbres citadinas, vinculándolas a sus expresiones culturales mediante las nuevas tendencias de la moda. No obstante, Becerra (2019) afirmó que empoderar a las mujeres con valores de ventas, mantiene una relación positiva con las ventas por catálogo. Asimismo, Massa (2018) sustentó que el reconocimiento a la política de igualdad de género, fortalece el portafolio de los lanzamientos de hasta 150 productos, lo que representa el 30\% de la facturación anual. Manzanares (2017) hizo una proyección al 2020, afirmando que de un total de 320 y 1000 mujeres serán capacitadas en tema de finanzas y ventas.

Las mujeres de la comunidad Shipiba en Lima permanecen como comunidad, resistiéndose a dejar su asentamiento a pesar de las constantes exigencias de desalojo y sin tener una vivienda propia, la explicación es que, hasta ahora, es el único lugar al que ellas han podido acceder como comunidad desde que llegaron a la gran ciudad y el Estado les cedió de forma momentánea, un espacio considerado como su hogar (Espinoza, 2019). Durante su estancia allí, reportan que han sido juzgadas y criticadas por no tener su vestimenta costumbrista, su especial modo de hablar el castellano y su manifestación cultural en la ciudad; sin embargo, eso las ha motivado a generar empresa en lo que saben hacer, confeccionar vestimentas con esencia Shipiba, accediendo al desarrollo conservando sus raíces (Vega, 2014).

León (1997) definió como poder o empoderamiento, a la facultad que tiene la persona con autoridad. El empoderamiento es el proceso mediante el cual un individuo fortalece las habilidades, la confianza, la percepción y el liderazgo a nivel social, aumenta el cambio de vida positivo y se vuelve independiente en la toma de decisiones. Libremente, es también una estrategia que incentiva a las mujeres a empoderarse, incrementar el uso y manejo de recursos materiales y simbólicos, influir y participar en el cambio social. De manera similar Rowlands (1997) cubre tres aspectos de la autoestima, la confianza en uno mismo y la personalidad, definidos como el desarrollo de las habilidades de un individuo. Estas relaciones cercanas representan la naturaleza de las relaciones y las decisiones y la capacidad de negociar 
e influir en las relaciones colectivas resultantes de la participación política cooperativa. Ahora bien, entender las dinámicas sociales de una comunidad nativa es un amplio abanico de muchas ventajas, pero al mismo tiempo se comprende en las perspectivas teóricas de la administración y las estrategias de marketing (Chiavenato, 2004; Kotler \& Armstrong, 2013). A nivel práctico se justifica en la comprensión de las dinámicas sociales de las comunidades nativa en cuanto a las ventas vestimentas con esencia Shipiba. Bajo este horizonte, la presente investigación describió los impactos socioeconómicos que ha tenido la implementación de estrategias de marketing para vender vestimentas con esencias shipibas en el presente contexto de pandemia COVID-19 en el mundo.

\section{MATERIALES Y MÉTODOS}

El presente estudio se basa en los resultados de investigaciones de docentes de la Universidad César Vallejo, Lima, realizadas desde los meses de setiembre 2020 a marzo de 2021 en la comunidad de Cantagallo, distrito del Rímac, Lima. Por lo cual, la intervención a la misma comunidad ha sido bajo los parámetros y protocolos de investigación de la Universidad y la autorización de los dirigentes y también del consentimiento informado de los participantes, respetando la confidencialidad de los mismos y los protocolos sanitarios de COVID-19.

Es un estudio con enfoque cualitativo (Flick, 2004). La visión que hace este tipo de investigación es integral, exigiendo la imparcialidad del investigador, empleando así un análisis exploratorio, indagando la naturaleza para proceder luego al discurso científico (Alvarez-Gayou, 2009, p. 24). En esa perspectiva, el investigador cualitativo estudia a las personas en el contexto de su pasado y de las situaciones próximas en las que suelen encontrarse, quiere descubrir algo novedoso, empleando en sí, la teoría fundamentada mediante la descripción de la realidad de modo complejo y holístico (Vasilaschis de Gialdino, 2006). Esa relación, dependerá mucho de la materialización de los hallazgos y observación más relevante (Ñaupas, et al., 2014, p.347). Por su naturaleza, se concatena con el paradigma de la teoría naturalista, caracterizada por una acción-reflexión-acción, ello implica que el investigador trate de generar un cambio y liberación de opresiones en un determinado contexto social (Guber, 2013). La población y muestra estuvo conformada por miembros de la comunidad Shipibo-Konibo que viven en Cantagallo, quienes provienen de la región Ucayali, la misma que fue el resultado de un proceso de poblamiento informal que actualmente está adherida arbitrariamente a la jurisdicción del distrito del Rímac (Matos, 2012). Se utilizó como instrumentos la entrevista, tomada con todas las medidas de salubridad en los tres primeros meses de 2021. 


\section{RESULTADOS}

El caso de las comunidades urbanas Shipibo Sus dinámicas de comercio se basan en productos textiles tradicionales de su cultura. Las ventas no solo les competen a las mujeres, también es responsabilidad de todos los miembros de la familia. Si bien es cierto, son las mujeres las expertas tejedoras, también los varones se involucran con los diseños y la pintura de las prendas. Ellos exhiben sus productos en plataformas virtuales para la venta; además de su habitual distribución en algunas zonas comerciales de Lima metropolitana.

Para el 2017, en esta comunidad se tenían 1493 habitantes Shipibo-Konibo (INEI, 2017), aunque se "ha incrementado hasta en un 4\% por natalidad y migración de las ciudades de Pucallpa y Tingo María” (Entrevista a Dirigente Shipibo, 2020). Una de las razones de tal incremento se visualiza en la asistencia de niños a la Escuela Intercultural Bilingüe. Adicionalmente del apoyo del Ministerio de Cultura que implementó un módulo de ventas y exhibición in situ para promocionar las vestimentas típicas que venía generando buenos resultados hasta antes de la pandemia, no obstante, en el contexto actual se hace imprescindible conectar dicha difusión a plataformas online, requiriéndose gerenciar un presupuesto para la sostenibilidad de dicho servicio, el mismo que podría ser mediante la implementación de préstamos a las familias emprendedoras con la finalidad que muevan sus capitales para seguir confeccionando prendas típicas: "Si bien hay apoyo por parte del Ministerio de Cultura, es necesario que el Estado nos incentive con préstamos a las familias. Con ese capital se generarán más ventas” (Entrevista a Poblador, 2021).

Los consumidores de esta moda mayoritariamente son extranjeros, los mismos que se interconectan con los vendedores mediante las plataformas virtuales, y redes sociales. De esa manera se garantiza la dinámica de las ventas online que, frente a una pandemia como la que se está viviendo, ha permitido reinventarse, como una medida de sobrevivencia para contrarrestar a la crisis causada por este azote viral. "Además de las ventas de vestimentas, las mascarillas con esencia Shipiba ha sido el mayor atractivo de la temporada” (Entrevista a Mujer Shipibo-Konibo, 2021).

Por otro lado, observando la organización de la comunidad como medio social, la gran mayoría de mujeres son jóvenes menores de 20 años, hijas de la primera generación de los migrantes que han asimilado la identidad cultural citadina, adaptándose a las nuevas tendencias de la moda urbana, generando así la simbiosis cultural que ahora sirve como una estrategia de marketing. Con la ayuda de éstas jóvenes, la comunidad de Cantagallo ha logrado entrar en la nueva concepción del Free Shipibo Style, una nueva tendencia de la moda actual, que ha fusionado rasgos urbanos sin perder la esencia 
Shipiba. Aunque con criterios diferentes, existen aún grupos que no favorecen la capacidad de respuesta que se espera de esta estrategia. Tal vez, con una mejor orientación de marketing se lograría la atención de un mayor público. Así, quizás si se utilizaran páginas web en lengua inglesa u otras lenguas extranjeras, las posibilidades de ventas serían mayores. Esta propuesta se refuerza en la evidencia observada en lo vivido por jóvenes Shipibos de las comunidades de Ucayali, quienes solamente han logrado exhibir sus productos, pinturas, artesanía, telares, etc. Las páginas web no resultaron lo suficientemente atrayentes como para generar una nueva concepción de compra dirigida a potenciales consumidores de las ciudades más grandes del mundo.

Zimmerman (2000) sostuvo que el empoderamiento es una guía útil para proponer metas, hitos y estrategias para implementar el cambio, así como un modelo teórico para proporcionar puntos de referencia y marcos para organizar el cambio. Sin embargo, para Charlier \& Caubergs (2007) el empoderamiento es el proceso de ganar primero poder individual y, posteriormente, de modo colectivo. En relación a la comunidad Shipibo- Konibo, es evidente que el trabajo individual no necesariamente hace referencia a una persona sino a una familia, pues, el pensamiento de comunidad centrada en la familia es una práctica ancestral (Manzanares, 2017), situación que podría transformarse en una ventaja por las extensiones de lazos consanguíneos que estas personas tienen dentro de la comunidad, reduciendo la comunidad a no más de 10 grandes familias. Entonces, al empoderar a una familia, es más sencillo empoderar al colectivo. Sin embargo, en el proceso se hace imprescindible seleccionar las actividades que cada uno de estos miembros realizaría, puesto que no todos deben hacer lo mismo, sino que deben desarrollar actividades complementarias acorde con sus aptitudes y su generación.

\section{DISCUSIÓN}

De los resultados cualitativos obtenidos se puede considerar estudios propicios como el da la ONU (2018) que consideran herramientas que brinden elementos prácticos y técnicos para empoderar para potenciar el empoderamiento económico con fines a practicar desarrollo sostenible en las mujeres, muchas de ellas de zonas rurales. La coyuntura de la pandemia ha permitido ha servido de plataforma para generar nuevos modelos de la moda, es el caso de las mascarillas con motivos Shipibos y también para desarrollar mayores nexos online de ventas potenciales, los mismos que son una poderosa herramienta de comercio (Villagomez, 2019). Por otra parte, para el empoderamiento de las mujeres, que desde la perspectiva de la comunidad Shipiba se traduciría en su competencia emprendedora, se hace necesario partir desde la especialización laboral ancestral, potencializándola dentro de su aptitud natural de fabricación de 
vestimentas y capacidad de comercialización, teniendo la ventaja competitiva natural de mercadeo (Cruz, 2015), considerando el marketing (Santos et al., 2017), como herramienta fundamental para el fomento de las ventas que se impulsan mediante las redes sociales (Castells, 2014) y la amplitud informativa que tiene en los ciudadanos que siempre están revisando sus equipos móviles Con esa ventaja, podría aprovecharse otra más, vinculada más hacia una mayor asiduidad del uso de dispositivos electrónicos en el grupo generacional más cercano a dichas tecnologías. Quienes serían los calificados para el manejo de las herramientas que permitiría mayor difusión y visualización de las ofertas hacia un público más numeroso, favoreciéndose así una mayor base de ventas y con ello, mayor dinamicidad económica a la comunidad. Lo que se busca en estas iniciativas de emprendimiento es que las mujeres ofrecen productos que reflejan siempre identidad con sus orígenes lo que les hace auténticos y únicos en sus producciones y ventas para la cual utilizan estrategias de marketing para facilitar su venta (Vega, 2014).

Se debe tener en cuenta, que, en un escenario de post pandemia, no se debe perder de vista las manifestaciones ancestrales de esta cultura viva, que, a pesar de mostrar mixturas interesantes, aún mantienen gran parte de su esencia cultural (Briceño, 2020). La esencia cultural su fuesen mejor orientadas, podría también transformase en una buena oportunidad para difundirla entre los interesados en aprender alguna de estas, como, por ejemplo, el idioma originario, gastronomía típica, expresiones musicales, ritos místicos; que en el contexto que vivimos siempre deben asumir políticas indígenas al estar en la ciudad como es caso de la comunidad urbanas Shipibo-Konibo establecida en la ciudad capital del Perú (Espinoza, 2016). Por otro lado, Briceño (2020) considera que las manifestaciones culturales de los grupos indígenas han sufrido influencias en la ciudad manteniendo su esencia, y adaptándose a las tendencias del mercado global.

En esa perspectiva, partir desde el empoderamiento de las mujeres resultaría una interesante estrategia, pues se tienen referencias muy buenas que demostraron relaciones positivas entre este proceso y las ventas por catálogo (Becerra, 2019). La apertura hacia un futuro debe superar los pensamientos retrógrados que se niega a reconocer la importancia de la política de igualdad de género (Massa, 2018). En una sociedad que cada día presenta mayores retos a los que las mujeres emprendedoras deben afrontar, ya con iniciativas propias, como las analizadas, para forjar un mejor mañana para ellas mismas y sus familias (Nussbaum \& Sen, 1998). 


\section{CONCLUSIONES}

La implementación de las estrategias de marketing para vender vestimentas con nuevas tendencias sin perder la esencia Shipiba mediante las redes sociales ha permitido empoderar a las mujeres de la Comunidad de Cantagallo y, sobre todo generar ingresos económicos para su bienestar personal y familiar, como también mejorar su calidad de vida. De manera que, el empoderamiento de las mujeres de la comunidad Shipiba es un claro modelo de emprendimiento para generar alternativas de mercado, basados en estrategias de marketing digital, mejoraron sus ventas utilizando herramientas tecnológicas informáticas.

Esto les ha permitido sobrevivir económicamente a la pandemia de la COVID-19. Por otro lado, con la participación de las jóvenes mujeres, la comunidad de Cantagallo ha logrado entrar en la nueva concepción del Free Shipibo Style, una nueva tendencia de la moda actual, que ha logrado fusionarse con rasgos urbanos sin perder la esencia Shipibo-Konibo. En este sentido, se recomienda que, para potencializar un mercado de vestimentas con esencia Shipiba, es necesario la implementación de publicidad en las redes sociales y/o plataformas virtuales, potencializando las competencias de los actores productivos acorde con sus aptitudes naturales, teniendo como unidad a la familia que, bajo su concepción cultural, se transformaría en el cimiento del empoderamiento comunitario ulterior.

\section{REFERENCIAS BIBLIOGRÁFICAS}

Álvarez-Gayou, J. (2009) Cómo hacer investigación cualitativa: fundamentos y metodología. México: Paidós

Alles, M (2008) Dirección estratégica de recursos humanos gestión por competencias. Gránica, México. https://bit.ly/33UxW8u

Becerra, Z. (2019) Propuesta para el empoderamiento de la mujer peruana en venta por catálogo Leonisa. Tesis para obtener el grado de maestra. Universidad César Vallejo, Lima.

Briceño Campos, P. (2020) Análisis del potencial turístico de las manifestaciones culturales inmateriales de la comunidad indígena shipiba de Cantagallo ubicada en la zona urbana del Rímac. Universidad César Vallejo.

Castells, M (2014) El poder de las redes sociales. La vanguardia Dossier, Madrid. https://bit.ly/3880yMX. 
Charlier, S. y Caubergs, L. (2007). El proceso de empoderamiento de las mujeres. Guía metodológica. Lovaina- Bélgica: Boone-Roosens.

Chiavenato, I. (2004) Introducción a la teoría general de la administración. Mc GrawHill.

Cuestas, A. (2010) Gestión del Talento humano y del conocimiento. 2da edición, ECOE.

Cruz, O (2015) El Empowerment y su relación con la satisfacción Laboral. Universidad Autónoma de Querétaro Facultad de Contaduría y Administración. México.

Espinoza, O. (2016) La política indígena en la ciudad. El caso de las comunidades urbanas ShipiboKonibo. En Surralles, A. Espinoza, O. y Jabin, D. Instituto Frances de Estudios Andinos. Lima.

Espinoza, O. (2019) La lucha por ser indígenas en la ciudad: el caso de la comunidad Shipibo-Konibo de Cantagallo en Lima. RIRA v. 4 (2): 153-184. https://doi.org/10.18800/revistaira.201902.005

Flick, U. (2004) Diseño de la Investigación Cualitativa. Ed. Morata, Madrid.

Guber, R. (2013) La articulación etnográfica descubrimiento y trabajo de campo en la investigación de Esther Hermitte. Buenos Aires; Biblos Culturalia.

Guevara Duarez, M., Tascca, S. C., Villanueva, P. P., del Aguila, J. S., \& Diaz, J. V. (2020). El aula invertida como metodología aplicada a estudiantes universitarios en el contexto covid-19. Revista Científica Pakamuros, 8(4), 3-14.

Hernandez, S. y Pulido, A. (2011) Fundamentos de gestión empresarial, enfoque basado en competencias. McGrawHill, México.

Instituto Nacional de Estadística e Informatica (2017) Censo Nacional, XII de Población y VII de Vivienda. Lima.

Kotler, P. y Armstrong, G. (2013) Fundamentos de marketing. 11e. Pearson Educación, México. ISBN 978-607-32-1722-4. https://bit.ly/3hMJram

León, M. (1997). Poder y empoderamiento de las mujeres. Bogotá- Colombia: Coedición de Tercer Mundo Editores, Vol. XI, N 18.

Ley $N^{\circ} 22175$ Ley de Comunidades nativas y de Desarrollo Agrario de la Selva y Ceja de Selva Li Y, Liu S, Zhang S, Ju Q, Zhang S, Yang Y, Wang H. (2020) Currenttreatment approaches for COVID19 and the clinical value of transfusion-related technologies, Transfusion and Apheresis Science. https://doi.org/10.1016/j.transci.2020.102839

Machicado, M. (2016) Mujeres empoderadas en Guatemala. En Organización de las Naciones UnidasMujeres (2016). Caja de Herramientas para el Empoderamiento Económico de las Mujeres Rurales Emprendedoras. Guatemala y el Salvador. https://bit.ly/3fOKbsM 
Manzanares, M. (2017). Belleza por un futuro, la iniciativa de L’Oréal para el empoderamiento económico de las mujeres peruanas. Diario Gestión.

Matos Mar, J. (2012) Perú, estado desbordado, la sociedad emergente nacional. Lima: Fondo editorial de la Universidad Ricardo Palma.

Massa, M. (2018). Belcorp, el negocio del empoderamiento femenino. Diario Gestión.

Nussbaum, M. y Sen, A. (1998) La Calidad de Vida, Fondo de Cultura Económica, México.

Ñaupas, H. Mejía, E. Novoa, E. Villagómez, A. (2014) Metodología de la investigación cuantitativa Cualitativa y redacción de la tesis. Ediciones de la U. Bogotá.

Organización de las Naciones Unidas-Mujeres (2016). Caja de Herramientas para el Empoderamiento Económico de las Mujeres Rurales Emprendedoras. Guatemala y el Salvador. https://bit.ly/3fOKbsM

Hernández, S. \& Pulido, A. (2011). Fundamentos de gestión empresarial: Enfoque basado en competencias. McGrawHill, México

Ramos, B. (2016) Mujeres empoderadas en: Organización de las Naciones Unidas-Mujeres (2016). Caja de Herramientas para el Empoderamiento Económico de las Mujeres Rurales Emprendedoras. Guatemala y el Salvador. https://bit.ly/3fOKbsM

Rowlands, J. (1997). Questioning empowerment. Oxford: Oxfam. https://bit.ly/2Ra8IjW

Santos, V., Spers, V., y Cremonezi, G. (2017). As Diferenças entre Marketing Multinível e as Pirâmides Financeiras ou “Esquema de Pirâmides”. Revista Brasileira de Marketing, 16(2), 243-251.

Vasilachis de Gialdino, I. (2006) Estrategias de investigación cualitativa. Gedisa Editorial. Barcelona. Vega, I. (2014). Buscando el Río: Identidad, Transformaciones y Estrategias de los Migrantes Indígenas Amazónicos en Lima Metropolitana. Centro Amazónico de Antropología y Aplicación Práctica. Vergara Causo, E., De la Cruz, M. Vertiz Osores, J., Fernandez Rosillo, F., Quispe, D. y Vertiz-Osores, R (2021) Gestión institucional y planificación estratégica en una institución educativa pública del distrito de Jaén, Cajamarca, Perú. Revista Pakamuros, 9 (1): 42-53. I: https://doi.org/10.37787/pakamuros-unj.v9i1.162

Vertiz-Osores, J.J., Guevara, M., Vilchez, G., Delgado, R., Cucho, R., y Vertiz, R. (2020) Tecnología para Atrapar el Agua: una experiencia exitosa y su impacto socioeconómico-ambiental. Revista Inclusiones 7 Abril/Junio ISSN 0719-4706. 
Villagomez, V. (2019) Ventas directas o de marketing marketing multinivel ¿Explotación de mujeres o camino hacia el Empoderamiento económico? Oikos Polis, Revista latinoamericana de Ciencias Económicas y Sociales, ISSN 2415-2250 ISSN 2521-960X 4 (1), 77-97.

Zimmerman, M. (2000). Teoría del empoderamiento. En J. Rappaport y E. Seidman (Eds). Manual de psicología comunitaria (pp. 43-63). Nueva York, Nueva York: Kluwer. 